\title{
Normalized single-shot X-ray absorption spectroscopy at a free-electron laser
}

\author{
Günter Brenner, ${ }^{1, *}$ (i) Siarhel Dziarzhytski, ${ }^{1}$ Piter S. Miedema, ${ }^{1}$ (i) Benedikt Rösner, ${ }^{2}$ (i) \\ Christian David, ${ }^{2}$ and Martin Beye ${ }^{1}$ (i) \\ ${ }^{1}$ Deutsches Elektronen Synchrotron DESY, Notkestr. 85, 22607 Hamburg, Germany \\ ${ }^{2} \mathrm{Paul}$ Scherrer Institut, $\mathrm{CH} 5232$ Villigen-PSI, Switzerland \\ *Corresponding author: guenter.brenner@desy.de
}

Received 27 February 2019; revised 25 March 2019; accepted 26 March 2019; posted 26 March 2019 (Doc. ID 359794$) ;$ published 17 April 2019

\begin{abstract}
A setup for dispersive X-ray absorption spectroscopy (XAS), employing a new reference scheme, has been implemented and tested at the soft X-ray free-electron laser (FEL) FLASH in Hamburg. A transmission grating was used to split the FEL beam into two copies (signal and reference). The spectral content of both beams was simultaneously measured for intensity normalization within the FEL bandwidth on a shot-to-shot basis. Excellent correlation between the two beams was demonstrated within a few percent for single bunch operation at $143 \mathrm{eV}$ photon energy. Applying the normalization scheme, an absorption spectrum of $\mathbf{a ~ G d}_{2} \mathrm{O}_{3}$ thin film sample was recorded around the $\mathrm{Gd} \mathrm{N}_{4,5}$-edge photon energy of $143 \mathrm{eV}$, showing excellent agreement with a reference spectrum measured at a synchrotron. This scheme opens the door for time-resolved single-shot XAS with femtosecond time resolution at FELs. () 2019 Optical Society of America
\end{abstract}

https://doi.org/10.1364/OL.44.002157

Provided under the terms of the OSA Open Access Publishing Agreement

X-ray absorption spectroscopy (XAS) is one of the most frequently applied spectroscopic X-ray techniques due to its experimental simplicity and large information content for a variety of fields composing material science, biology, and chemistry [1-3]. XAS provides element-specific information on the electronic structure which encodes the geometry, symmetries, chemical surrounding, and spin configurations of the interrogated element $[4,5]$. Moreover, it has been shown that timeresolved XAS can track ultrafast nuclear and electronic structure dynamics during chemical reactions [6-8]. Although FELs provide femtosecond X-ray pulses at an unprecedented photon flux [9-11] ideal to study such ultrafast processes, the application of time-resolved XAS at free-electron lasers (FELs) with femtosecond time resolution is extremely challenging [12]. The reason is that most FELs today are based on the self-amplified spontaneous emission (SASE) process [13]. Due to the stochastic nature of the SASE process, the inherent strong shot-to-shot fluctuations both in intensity and spectral composition lead to a constantly changing spectral fingerprint of the incident beam [14]. At the soft x-ray FEL FLASH the photon pulses exhibit a relative total energy bandwidth of typically $\mathrm{dE} / \mathrm{E} \approx 0.5-1 \%$ containing typically up to about 20 narrow spikes $[15,16]$. To achieve sufficient energy resolution for XAS, the use of a monochromator is required, which greatly amplifies intensity fluctuations, since one of the spikes may or may not hit the chosen energy window. Thus, in order to obtain a quantitative $\mathrm{x}$-ray absorption spectrum, an efficient and proper intensity normalization scheme that would be linear over a large dynamic range is required.

In this Letter, we report on the implementation of a dispersive XAS method at the PG2 monochromator beamline [17] at FLASH, making use of an advanced reference scheme for normalization. Similar to the approach realized at SACLA [18] and LCLS [19] it employs a diffractive transmission grating (TG) with low line density, where the positive and negative first diffraction orders are used to provide two identical copies of the pulse for single-shot intensity normalization for each spectral sample. While one beam travels through the sample, the other is used as an intensity reference. Alternatively, for time-resolved experiments, both extreme ultraviolet beams can also be transmitted through the sample, while only the interaction point with one of the beams is overlapped with the optical femtosecond pump-probe laser of FLASH [20]. This allows for a proper normalization of pump-laser induced changes of the absorption. Studies of signal and reference beam intensity correlation for different energy bandwidths and photon pulse numbers show excellent correspondence. The potential for XAS is demonstrated for a gadolinium oxide $\left(\mathrm{Gd}_{2} \mathrm{O}_{3}\right)$ thin film at one of the gadolinium $(\mathrm{Gd}) \mathrm{N}_{4,5}$-edge pre-resonances and can be readily extended to perform femtosecond time-resolved XAS (TR-XAS) at FLASH.

A set of diffractive TGs is installed at the PG2 beamline branch behind the monochromator, which disperses the incoming FEL light in the vertical direction (Fig. 1). The monochromatized FEL radiation is then diffracted in a horizontal direction by the TG. Its diffraction orders enter the permanently installed split-and-delay unit (SDU), [21] which, in this case, is solely used to steer the signal and reference beams independently through the beamline towards the experimental setup, such that they are parallel and separated well enough to allow for inserting a sample into one beam only. Depending on 


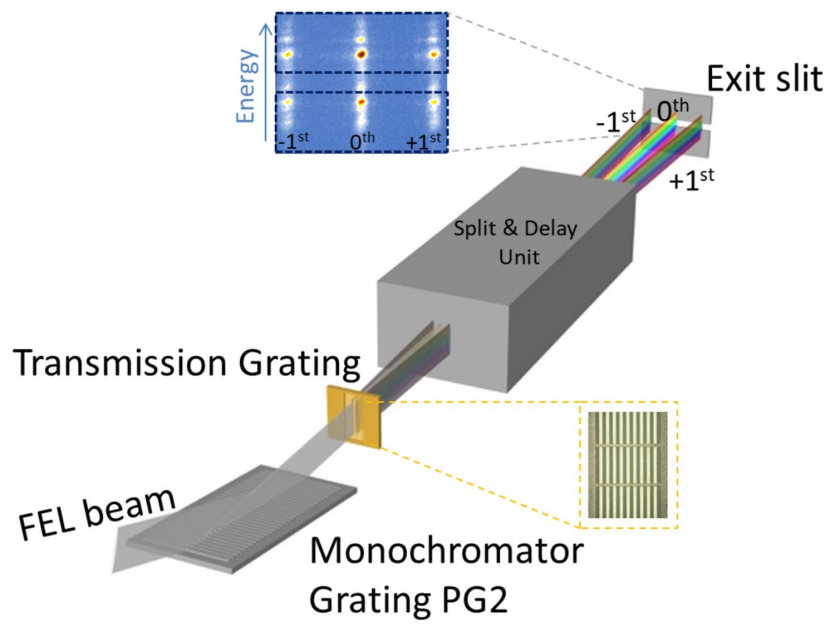

Fig. 1. Schematic of the setup for XAS at FLASH using a TG to split the FEL beam into a signal and reference beam $(+1$ st and -1 st order, respectively).

the requirements, different diffraction orders of the TGs can be transported: either the equal intensity 1 st order beams $(+1 /-1)$ or the more intense 0th-order beam, together with one of the 1st-order beams to compensate for sample absorption to yield similar intensities on the detector. Herein, we show data recorded with an intensified CCD imaging the visible light fluorescence induced by both beams on a Ce:YAG screen in the dispersion plane of the monochromator. We transported the beams to the beamline focus position, where we achieved horizontal separations of several millimeters, limited by the aperture of the differential pumping unit integrated at the end of the beamline.

The diffractive TGs were installed on a manipulator five meters downstream of the monochromator. Their periodicity was chosen as $34 \mu \mathrm{m}$, meeting the geometrical requirements for sufficient beam separation while fitting the optical aperture of the beamline. Nine grating bars provide a horizontal aperture size of $0.3 \mathrm{~mm}$, whereas the vertical size was limited to $4 \mathrm{~mm}$ by the substrate size. For fabrication of the TGs, a $4 \times 4 \mathrm{~mm}$ large silicon nitride membrane was coated with a conductive metal layer and a lithography resist. After exposure in an electron lithography tool (Vistec EBPG 5000+) and development of the exposed parts, a thin gold film was electrochemically deposited. In a subsequent lithography step, support structures were deposited to ensure the mechanical stability of the grating. Finally, the silicon nitride membrane on the backside was removed in a reactive ion etching step. The resulting structure is a $100 \mathrm{~nm}$ thick, free-standing grating with a theoretical $+/-1$ st-order diffraction efficiency exceeding $20 \%$ at $143 \mathrm{eV}$.

The top of Fig. 2 shows the relative intensity ratio between the 0th-order and $+/-1$ st order diffraction of the TG measured at a photon energy of $143 \mathrm{eV}$ at the monochromator exit plane [22] without using the SDU. The ratio of each SASE spike in the 1 st and 0 th diffraction orders is determined as approximately 0.6 . This compares to a measured 1 st-order diffraction efficiency of $14 \%$. The central image in Fig. 2 shows a single shot of FLASH, where the three beams are separated by the TG in the horizontal direction, while the vertical dispersion plane of the high-resolution monochromator resolves the

\section{Diffraction orders}

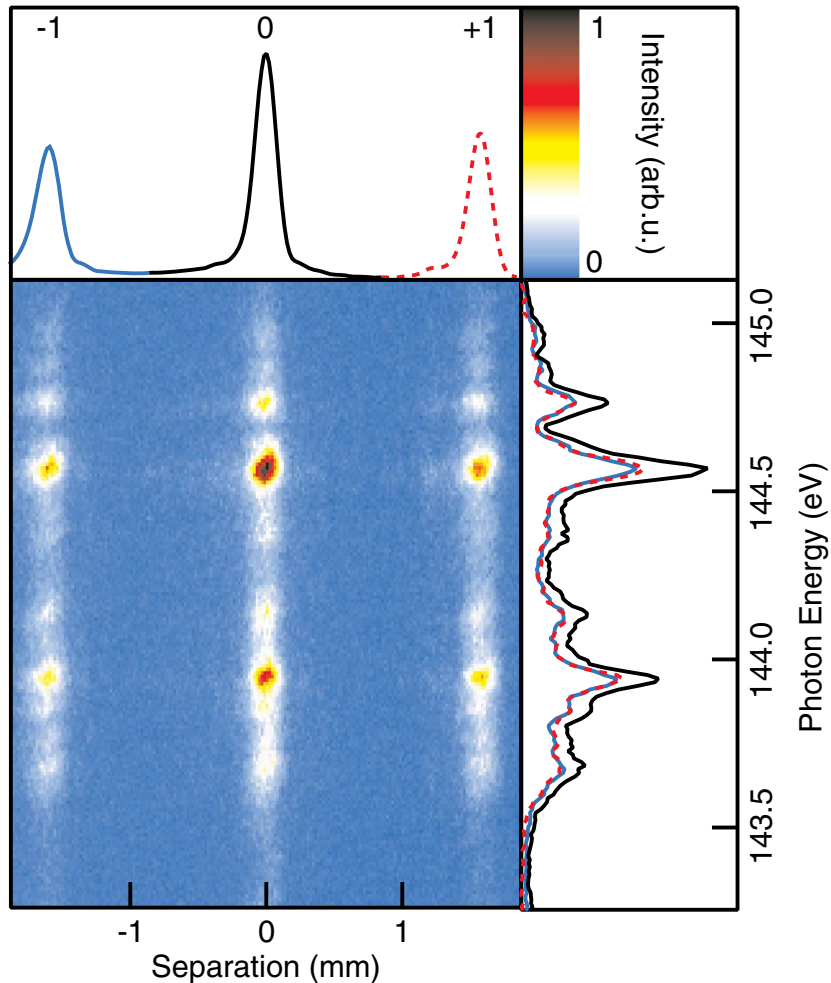

Fig. 2. Top: diffraction efficiency of the TG. Each first diffraction order contains about $60 \%$ of the intensity as compared to the 0thorder beam. Bottom, left: dispersed single shot from FLASH behind the TG. In the dispersion plane of the PG monochromator, the vertically dispersed spiky SASE spectral distribution split by the TG into the three described orders is shown. The horizontal separation of approximately $2 \mathrm{~mm}$ is determined by the TG design. Bottom, right: the spectral distributions for this shot of the three orders. The black line shows the dominant 0th-order beam, while the overlapping red and blue lines display the spectral distribution in the 1 st-order beams.

modes in the SASE spectrum. On the right, we display the spectra of each of the three beams, using the same color coding as in the top panel. The spectra of both the +1 st (red dashed line) and -1 st (blue line) diffraction order show the strong correlation in intensity and energy between these positive and negative diffraction orders.

In Fig. 3, we display the intensity correlation in both arms by showing the integral intensity in each of the 1st-order beams. On the left, we integrate over an energy window of $10 \mathrm{meV}$ and obtain a Pearson correlation coefficient of $99.1 \%$ across 3000 individual shots. Averaging over a $1 \mathrm{eV}$ energy window increases the Pearson correlation coefficient of this data set to $99.9 \%$, as shown on the right.

In order to estimate how much acquisition time is needed in order to get a certain sensitivity to changes in the XAS spectrum at a given energy bandwidth, we analyze the plots presented in Fig. 3 by fitting a line to the single-shot correlation data. The slope of the line then directly reflects the intensity ratio of both arms, i.e., the transmission of a sample placed into one $\mathrm{arm}$. The zero crossing of this fit is a measure of the different background values in each arm. We consider the relative error (one standard deviation) of the fitted slope as an indicator for 




Fig. 3. Intensity correlation between the signal and reference beams. (a) Total spectral intensity in each of the 1st-order beams is integrated over a $10 \mathrm{meV}$ energy window, showing a correlation of $99.1 \%$ (Pearson correlation coefficient) across 3000 shots. (b) Increasing the integration window to $1 \mathrm{eV}$, the correlation coefficient increases to $99.9 \%$.

the sensitivity of the measurement. Figure 4 shows this value color-coded for acquiring multiple pulses and for a given energy bandwidth around $143 \mathrm{eV}$ on logarithmic axes. For example, if the required energy bandwidth is $0.1 \mathrm{eV}$, we derive that averaging the spectra of 60 shots is sufficient to get below $1 \%$ relative error in transmission measurements. To reach below the $0.1 \%$ level, about 5000 pulses are required, with an acquisition time as small as one second only, as FLASH produces up to 500 photon pulses per burst at a repetition rate of $10 \mathrm{~Hz}$. Increasing the measurement time by averaging even more shots and increasing the averaged energy bandwidth, improves the sensitivity into the per mille level, as can be seen in Fig. 4.

To demonstrate the applicability of this normalization scheme to a XAS measurement, a $30 \mathrm{~nm}$ thick film of $\mathrm{Gd}_{2} \mathrm{O}_{3}$ deposited on a $200 \mathrm{~nm}$ parylene $\mathrm{N}$ membrane provided by Luxel Corp. was placed into one copy of the beam. The diffracted beam orders, one with a sample and one without it as reference, were projected side-by-side onto the detector. This scheme allows employing the full bandwidth of FLASH for

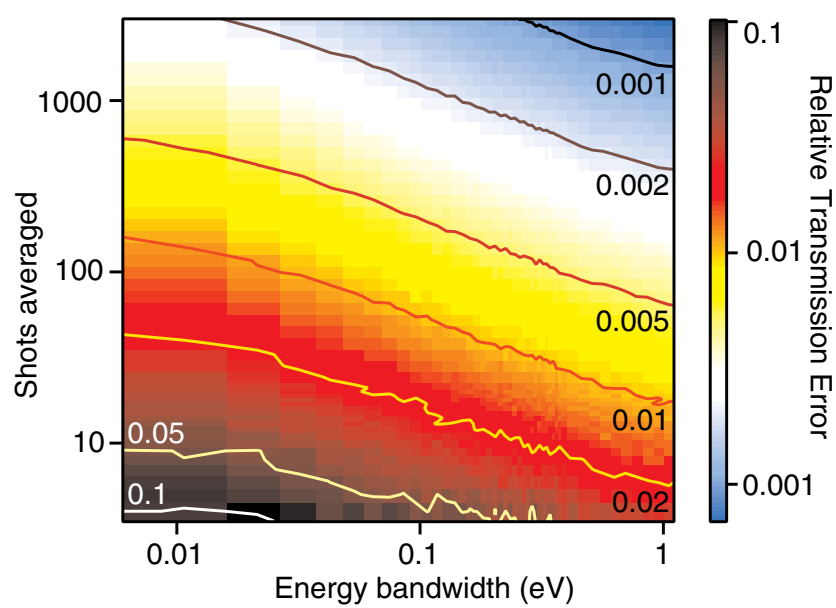

Fig. 4. Sensitivity to spectral changes for different integration windows in energy and measurement time. The correlation plots of Fig. 3 are fitted with a line. The fit error of the slope is taken as an indicator for the sensitivity to changes in the transmission measurement.

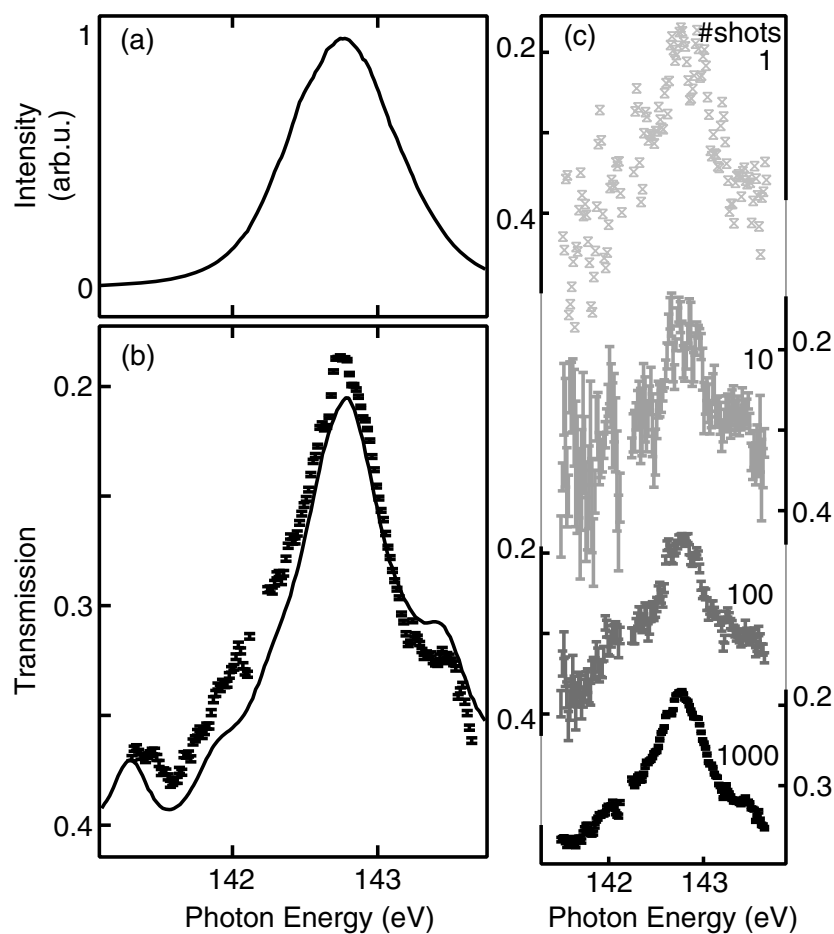

Fig. 5. (a) Average spectral distribution of FLASH. A total energy bandwidth of $0.9 \mathrm{eV} \mathrm{FWHM}$ was measured. (b) $\mathrm{Gd} \mathrm{N}_{4,5}$-edge $\mathrm{x}$-ray absorption spectrum measured in transmission of a $\mathrm{Gd}_{2} \mathrm{O}_{3}$ thin film. The symbols represent data recorded at FLASH, while the solid line displays a reference measurement of the sample from a synchrotron. (c) Single-shot and averaged spectra for 10, 100, and 1000 FEL pulses.

each photon pulse. Figure 5 displays the recorded data as a function of energy. The averaged spectral intensity distribution of FLASH is depicted in Fig. 5(a) and shows a full width at halfmaximum (FWHM) bandwidth of about $0.9 \mathrm{eV}(\approx 0.6 \%)$ as expected. The inital FEL pulse duration was around $100 \mathrm{fs}$ (FWHM), which was elongated to $\approx 180$ fs (FWHM) due to the pulse stretching of the grating monochromator. The FEL pulse energy was determined as $5 \mathrm{uJ}$ with $1 \mathrm{~mm}$ apertures used in the photon diagnostics section before the PG2 beamline. The extracted transmission spectrum is shown in the bottom left panel of Fig. 5 (symbols with one standard deviation error bars) and compared with a Gd $\mathrm{N}_{4,5}$-edge XAS measurement recorded at the PM3 beamline of the synchrotron BESSY-II (solid line). At FLASH, the monochromator dispersion was set to $800 \mathrm{meV} / \mathrm{mm}$, which is dispersed by $15.6 \mathrm{meV}$ per pixel on the camera. Within the bandwidth of FLASH, the agreement is excellent, and small differences can be attributed to potential inhomogeneities of the sample or changes in the sample composition during storage and transport between the facilities under ambient atmosphere.

In summary, we demonstrated a powerful scheme to normalize FEL intensities with high sensitivity, dividing the beam with a TG. This scheme is integrated into the beamline PG2 at FLASH and, thus, can make use of its capabilities to monochromatize and focus the beam, as well as to conduct pumpprobe XAS experiments with femtosecond time resolution. Its specific advantage lies in the possibility to conduct spectroscopy using the full SASE bandwidth of FLASH on a shot-to-shot 
basis-without scanning the monochromator. The demonstrated high spectral quality and sensitivity to small changes, in combination with the high repetition rate of FLASH, provides ideal conditions to map out large parameter spaces, varying, e.g., pump-probe delay, pump intensity, and wavelength, as well as probe intensity and wavelength. This provides the crucial step towards finally making XAS a standard tool at FELs.

Funding. Helmholtz Association (VH-NG-110, VH-NG1105); European Union (EU-H2020) (654360).

Acknowledgment. The authors gratefully acknowledge the support of the scientific and technical team at FLASH. We acknowledge Christian Schüssler-Langeheine and Daniel Schick for their support in measuring the synchrotron reference spectrum.

\section{REFERENCES}

1. J. Stöhr, NEXAFS Spectroscopy (Springer, 1992).

2. B. Watts and H. Ade, Mater. Today 15(4), 148 (2012).

3. F. M. F. de Groot and A. Kontani, Core Level Spectroscopy of Solids (CRC Press, 2008).

4. X. Zhang, T. Palamarciuc, P. Rosa, J.-F. Létard, B. Doudin, Z. Zhang, J. Wang, and P. A. Dowben, J. Phys. Chem. C 116, 23291 (2012).

5. B. Rösner, M. Milek, A. Witt, B. Gobaut, P. Torelli, R. H. Fink, and M. M. Khusniyarov, Angew. Chem. (Int. Ed.) 54, 12976 (2015).

6. P. Wernet, K. Kunnus, I. Josefsson, I. Rajkovic, W. Quevedo, M. Beye, S. Schreck, S. Grübel, M. Scholz, D. Nordlund, W. Zhang, R. W. Hartsock, W. F. Schlotter, J. J. Turner, B. Kennedy, F. Hennies, F. M. F. de Groot, K. J. Gaffney, S. Techert, M. Odelius, and A. Föhlisch, Nature 520, 78 (2015).

7. N. Huse, H. Cho, K. Hong, L. Jamula, F. M. F. de Groot, T. K. Kim, J. K. McCusker, and R. W. Schoenlein, J. Phys. Chem. Lett. 2, 880 (2011).

8. C. Bressler, C. Milne, V.-T. Pham, A. EINahhas, R. M. van der Veen, W. Gawelda, S. Johnson, P. Beaud, D. Grolimund, M. Kaiser, C. N. Borca, G. Ingold, R. Abela, and M. Chergui, Science 323, 489 (2009).

9. W. Ackermann, G. Asova, V. Ayvazyan, A. Azima, N. Baboi, J. Bähr, V. Balandin, B. Beutner, A. Brandt, A. Bolzmann, R. Brinkmann, O. I. Brovko, M. Castellano, P. Castro, L. Catani, E. Chiadroni, S. Choroba, A. Cianchi, J. T. Costello, D. Cubaynes, J. Dardis, W. Decking, H. Delsim-Hashemi, A. Delserieys, G. Di Pirro, M. Dohlus, S. Düsterer, A. Eckhardt, H. T. Edwards, B. Faatz, J. Feldhaus, K. Flöttmann, J. Frisch, L. Fröhlich, T. Garvey, U. Gensch, C. Gerth, M. Görler, N. Golubeva, H. Grabosch, M. Grecki, O. Grimm, K. Hacker, U. Hahn, J. H. Han, K. Honkavaara, T. Hott, M. Hüning, Y. Ivanisenko, E. Jaeschke, W. Jalmuzna, T. Jezynski, R. Kammering, V. Katalev, K. Kavanagh, E. T. Kennedy, S. Khodyachykh, K. Klose, V. Kocharyan, M. Körfer, M. Kollewe, W. Koprek, S. Korepanov, D. Kostin, M. Krassilnikov, G. Kube, M. Kuhlmann, C. L. S. Lewis, L. Lilje, T. Limberg, D. Lipka, F. Löhl, H. Luna, M. Luong, M. Martins, M. Meyer, P. Michelato, V. Miltchev, W. D. Möller, L. Monaco, W. F. O. Müller, O. Napieralski, O. Napoly, P. Nicolosi, D. Nölle, T. Nuñez, A. Oppelt, C. Pagani, R. Paparella, N. Pchalek, J. Pedregosa-Gutierrez, B. Petersen, B. Petrosyan, G. Petrosyan, L. Petrosyan, J. Pflüger, E. Plönjes, L. Poletto, K. Pozniak, E. Prat, D. Proch, P. Pucyk, P. Radcliffe, H. Redlin, K. Rehlich, M. Richter, M. Roehrs, J. Roensch, R. Romaniuk, M. Ross, J. Rossbach, V. Rybnikov, M. Sachwitz, E. L. Saldin, W. Sandner, H. Schlarb, B. Schmidt, M. Schmitz, P. Schmüser, J. R. Schneider, E. A. Schneidmiller, S. Schnepp,
S. Schreiber, M. Seidel, D. Sertore, A. V. Shabunov, C. Simon, S. Simrock, E. Sombrowski, A. A. Sorokin, P. Spanknebel, R. Spesyvtsev, L. Staykov, B. Steffen, F. Stephan, F. Stulle, H. Thom, K. Tiedtke, M. Tischer, S. Toleikis, R. Treusch, D. Trines, I. Tsakov, E. Vogel, T. Weiland, H. Weise, M. Wellhöfer, M. Wendt, I. Will, A. Winter, K. Wittenburg, W. Wurth, P. Yeates, M. V. Yurkov, I. Zagorodnov, and K. Zapfe, Nat. Photonics 1, 336 (2007).

10. P. Emma, R. Akre, J. Arthur, R. Bionta, C. Bostedt, J. Bozek, A. Brachmann, P. Bucksbaum, R. Coffee, F. J. Decker, Y. Ding, D. Dowell, S. Edstrom, A. Fisher, J. Frisch, S. Gilevich, J. Hastings, G. Hays, P. Hering, Z. Huang, R. Iverson, H. Loos, M. Messerschmidt, A. Miahnahri, S. Moeller, H. D. Nuhn, G. Pile, D. Ratner, J. Rzepiela, D. Schultz, T. Smith, P. Stefan, H. Tompkins, J. Turner, J. Welch, W. White, J. Wu, G. Yocky, and J. Galayda, Nat. Photonics 4, 641 (2010).

11. T. Ishikawa, H. Aoyagi, T. Asaka, Y. Asano, N. Azumi, T. Bizen, H. Ego, K. Fukami, T. Fukui, Y. Furukawa, S. Goto, H. Hanaki, T. Hara, T. Hasegawa, T. Hatsui, A. Higashiya, T. Hirono, N. Hosoda, M. Ishii, T. Inagaki, Y. Inubushi, T. Itoga, Y. Joti, M. Kago, T. Kameshima, H. Kimura, Y. Kirihara, A. Kiyomichi, T. Kobayashi, C. Kondo, T. Kudo, H. Maesaka, X. M. Maréchal, T. Masuda, S. Matsubara, T. Matsumoto, T. Matsushita, S. Matsui, M. Nagasono, N. Nariyama, H. Ohashi, T. Ohata, T. Ohshima, S. Ono, Y. Otake, C. Saji, T. Sakurai, T. Sato, K. Sawada, T. Seike, K. Shirasawa, T. Sugimoto, S. Suzuki, S. Takahashi, H. Takebe, K. Takeshita, K. Tamasaku, H. Tanaka, R. Tanaka, T. Tanaka, T. Togashi, K. Togawa, A. Tokuhisa, H. Tomizawa, K. Tono, S. Wu, M. Yabashi, M. Yamaga, A. Yamashita, K. Yanagida, C. Zhang, T. Shintake, H. Kitamura, and N. Kumagai, Nat. Photonics 6, 540 (2012).

12. D. P. Bernstein, Y. Acremann, A. Scherz, M. Burkhardt, J. Stöhr, M. Beye, W. F. Schlotter, T. Beeck, F. Sorgenfrei, A. Pietzsch, W. Wurth, and A. Föhlisch, Appl. Phys. Lett. 95, 134102 (2009).

13. B. W. J. McNeil and N. R. Thompson, Nat. Photonics 4, 814 (2010).

14. E. L. Saldin, E. A. Schneidmiller, and M. V. Yurkov, New J. Phys. 12, 035010 (2010).

15. S. Düsterer, M. Rehders, A. Al-Shemmary, C. Behrens, G. Brenner, O. Brovko, M. DellAngela, M. Drescher, B. Faatz, J. Feldhaus, U. Frühling, N. Gerasimova, N. Gerken, C. Gerth, T. Golz, A. Grebentsov, E. Hass, K. Honkavaara, V. Kocharian, M. Kurka, T. Limberg, R. Mitzner, R. Moshammer, E. Plönjes, M. Richter, J. Rönsch-Schulenburg, A. Rudenko, H. Schlarb, B. Schmidt, A. Senftleben, E. A. Schneidmiller, B. Siemer, F. Sorgenfrei, A. A. Sorokin, N. Stojanovic, K. Tiedtke, R. Treusch, M. Vogt, M. Wieland, W. Wurth, S. Wesch, M. Yan, M. V. Yurkov, H. Zacharias, and S. Schreiber, Phys. Rev. Spec. Top.—Accel. Beams 17, 120702 (2014).

16. S. Krinsky and R. L. Gluckstern, Phys. Rev. Spec. Top.-Accel. Beams 6, 050701 (2003).

17. M. Martins, M. Wellhöfer, J. T. Hoeft, W. Wurth, J. Feldhaus, and R. Follath, Rev. Sci. Instrum. 77, 115108 (2006).

18. T. Katayama, Y. Inubushi, Y. Obara, T. Sato, T. Togashi, K. Tono, T. Hatsui, T. Kameshima, A. Bhattacharya, Y. Ogi, N. Kurahashi, K. Misawa, T. Suzuki, and M. Yabashi, Appl. Phys. Lett. 103, 131105 (2013).

19. W. F. Schlotter, "Ultimate spectroscopic sensitivity with balanced x-ray absorption" (to be published).

20. H. Redlin, A. Al-Shemmary, A. Azima, N. Stojanovic, F. Tavella, I. Will, and S. Düsterer, Nucl. Instrum. Methods Phys. Res., Sect. A 635, S88 (2011).

21. F. Sorgenfrei, W. F. Schlotter, T. Beeck, M. Nagasono, S. Gieschen, H. Meyer, A. Föhlisch, M. Beye, and W. Wurth, Rev. Sci. Instrum. 81, 043107 (2010).

22. N. Gerasimova, S. Dziarzhytski, and J. Feldhaus, J. Mod. Opt. 58, 1480 (2011). 